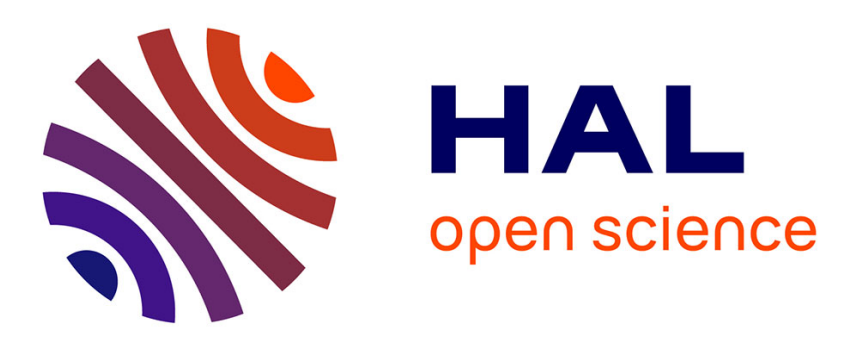

\title{
Preferential Growth of Force Network in Granular Media
}

Mehdi Pouragha, Jérôme Duriez, Antoine Wautier, Richard Wan, François

Nicot, Félix Darve

\section{To cite this version:}

Mehdi Pouragha, Jérôme Duriez, Antoine Wautier, Richard Wan, François Nicot, et al.. Preferential Growth of Force Network in Granular Media. Granular Matter, 2019, 21 (3), pp.14. 10.1007/s10035019-0919-1 . hal-02172048

\section{HAL Id: hal-02172048 \\ https://hal.science/hal-02172048}

Submitted on 3 Jul 2019

HAL is a multi-disciplinary open access archive for the deposit and dissemination of scientific research documents, whether they are published or not. The documents may come from teaching and research institutions in France or abroad, or from public or private research centers.
L'archive ouverte pluridisciplinaire HAL, est destinée au dépôt et à la diffusion de documents scientifiques de niveau recherche, publiés ou non, émanant des établissements d'enseignement et de recherche français ou étrangers, des laboratoires publics ou privés. 


\title{
Preferential Growth of Force Network in Granular Media
}

\author{
Mehdi Pouragha ${ }^{1} \quad$ Jérôme Duriez ${ }^{2} \quad$ Antoine Wautier ${ }^{2}$ \\ Richard Wan ${ }^{1} \quad$ François Nicot ${ }^{3} \quad$ Félix Darve $^{4}$ \\ ${ }^{1}$ Civil Engineering Department, University of Calgary, Calgary, \\ Canada \\ ${ }^{2}$ Irstea, Aix Marseille University, RECOVER, Aix-en-Provence, \\ France \\ ${ }^{3}$ Irstea, Grenoble Alpes University, Grenoble, France \\ ${ }^{4}$ Laboratoire 3SR, Grenoble Alpes University, Grenoble,France
}

\begin{abstract}
The paper studies the evolution of contact forces in granular materials as a preferential growth process whereby the incremental change in forces is proportional to their current values. Preferential growth of forces is depicted herein as a positive feedback process that enhances the heterogeneity in granular media over the course of loading. As an initial step, $2 \mathrm{D}$ discrete element simulations are performed to verify the hypothesis in the simpler case of hydrostatic loading, with an extension to deviatoric loading thereafter suggested. Not being predicated upon particularities of any mechanical model, the preferential growth mechanism reveals the commonality between the force network and other self-organized complex systems with dynamic interaction networks. The paper concludes with potential mechanical consequences such a dependency between current state and the incremental change entails.
\end{abstract}

\section{Introduction}

The advent of photoelastic experimental techniques $[1,2]$ and discrete element methods [3] in the 1970's opened a new window into the microscopic features of contact network and force transmission within granular media. The pioneering experiments of Bob Behringer and coworkers $[4,5]$ further visualized the way the stress applied on the boundaries is transmitted through an intricate network of force chains. Intriguingly, the structure of force transmission in granular media highly resembles other self-organized networks encountered in nature, see Fig. 1. More interestingly, the force network and its evolution in confined granular media share many statistical characteristics with other complex systems that 
involve a multitude of evolving interactions [6], such as social and economic systems, and traffic, see e.g. [7] for more detailed juxtapositions.

Although the development of force network patterns can be aptly understood and reproduced by mechanical analysis and numerical simulations such as molecular and contact dynamic algorithms, nevertheless, the resemblance between the force network in granular media and other seemingly unrelated systems suggests a deeper mutual underlying physics that transcends the particular governing (constitutive) laws for each system. The field of network science partially addresses this question of common characteristics of various systems by providing a proper substrate-neutral depiction of interconnected multi-agent systems.

"Preferential attachment" is among the most prominent class of processes that is recognized in a variety of different complex systems and dynamic networks with evolving internal structure. Coined first in [8], the term preferential attachment, in its general form, describes phenomena in which a change in the system's state is distributed among its constituents proportionally to their current state. The basic concept of preferential evolutions has indeed been observed in different fields with its statistical representation tracing back to classical works such as the Pólya urn model [9] and the so-called Yule processes [10]. In sociology, the same process is sometimes known as the Matthew effect [11] which encapsulates the common idea of "rich gets richer".

In simple words, the logic behind such preferential evolutions can be described as follows. During a given process, if, due to some persisting "cause", a non-uniform pattern of distribution of a variable develops in a system, then, due to the same cause, an additional change in the system is likely to follow the current distribution of state; i.e. larger current values receive yet a larger portion of increment and vice versa. Hence, the preferential evolution transcends the governing laws of different systems in that its realization is not predicated upon our knowledge of the above-mentioned cause.

Therefore, contrary to diffusion processes which often restore homogeneity via a negative feedback, preferential growth by contrast enhances heterogeneity through a positive feedback where the disparity between the lowest and highest values in the system continuously increases as the process unfolds.

Coming back to granular media, the photoelastic experiments by Bob Behringer and co-workers [12] show that, while for soft particles, the distribution of contact forces is relatively diffused, a persistent structure in force network rapidly develops for stiff particles. The formation of such long-lasting structures can indeed be explained in terms of a preferential growth process that bypass particularities of mechanical considerations at both micro- and macro-levels.

From such a perspective, the present study explores the evolution of interparticle forces in granular media in relation to the existing configuration of force network. By employing 2D Discrete Element Method (DEM) simulations, the incremental change in contact forces is systematically tracked during both hydrostatic and deviatoric loading paths. Interestingly, the evolution of forces is shown to follow a preferential growth rule whereby the increment of change is proportional to the current value of the contact force. For the deviatoric- 

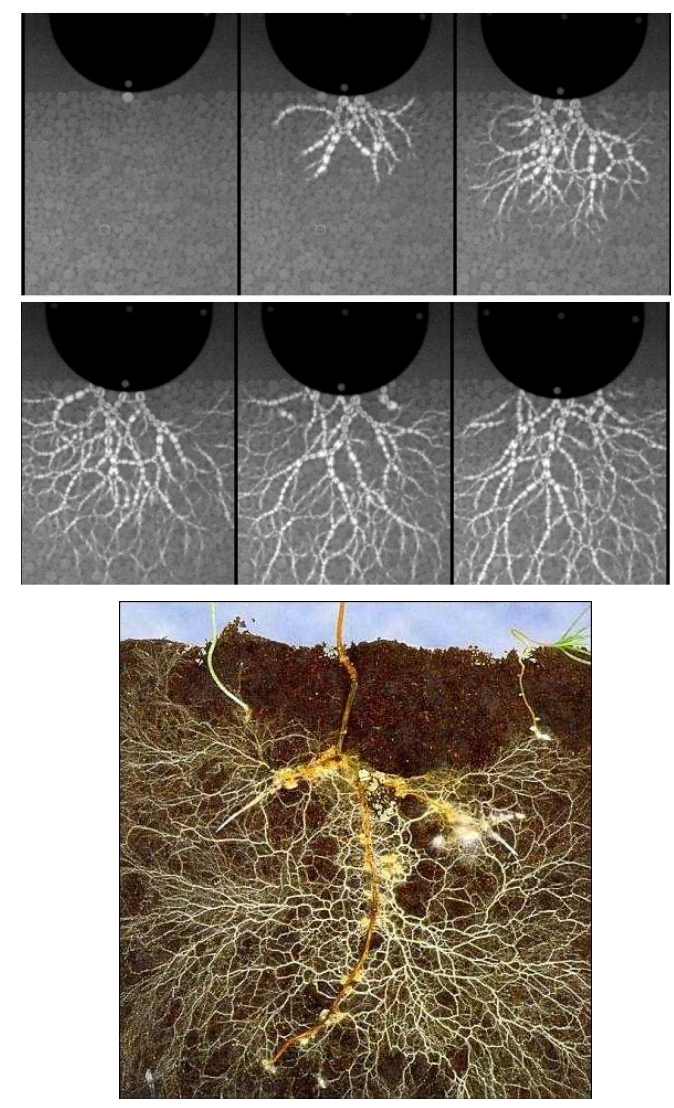

Figure 1: (Top) Network of interparticle forces in granular materials in a photoelastic experiment, adopted from [12], (Bottom) Mycorrhizal network of plants roots (Copyright of Odair Alberton, Universidade Paranaense, Brazil. Used with permission). 
here, isobaric - loading path, an extension of the general rule is shown to hold independently along principal stress directions. When integrated, the preferential growth rule projects a linear variation of contact forces with relevant stress variables, which is also confirmed by numerical simulations. While not delved into deeply in this brief communication, such a dependency between incremental changes and the current state can have fundamental repercussions in the mechanical understanding of granular media, and can also be employed to elaborate the interdependencies among the various local variables at play [13, 14].

\section{Preferential Growth of Forces: A Simple Case of Hydrostatic Stress}

For a process evolving according to the preferential growth rule, the incremental change in the targeted local variable is proportional to its current value. In other words, the global change is distributed locally, proportional to the current local distribution. It is worth mentioning that the analysis in this section is partly inspired by the graph theoretical analysis for evolving networks offered in [15], among others. For the simpler case of hydrostatic loading in granular materials, the preferential growth hypothesis predicts a general evolution relation of the following form:

$$
d f^{c} \propto f^{c} \quad \text { for all contacts } c
$$

where the $f^{c}$ and $d f^{c}$ are the current value and the change in the magnitude of the force at contact $c$, respectively, and the relation is assumed to hold in a statistical sense for all the contacts. Assuming the validity of the hypothesis in Eq. 1, a more explicit form of the relation can be written for the hydrostatic loading where the change in the forces is due to a change in hydrostatic pressure, $p$, i.e.

$$
\frac{d f^{c}}{d p}=A \frac{f^{c}}{\sum_{\alpha=1}^{N_{c}} f^{\alpha}}
$$

with $A$ being a proportionality coefficient with dimension of a length in $2 \mathrm{D}$, and $N_{c}$ the number of contacts. Here, the coefficient $A$ is taken to be the same for all the contacts, which renders Eq. 2 non-trivial.

Moreover, the summation in the denominator runs over all the contacts and represents merely a normalizing constant that turns the current value of a particular contact force into a weighting function which further facilitates subsequent calculations. Summing the two sides of Eq. 2 over all contacts leads to:

$$
\frac{\sum_{\alpha=1}^{N_{c}} d f^{\alpha}}{d p}=A \frac{\sum_{\alpha=1}^{N_{c}} f^{\alpha}}{\sum_{\alpha=1}^{N_{c}} f^{\alpha}} \rightarrow \sum_{\alpha=1}^{N_{c}} d f^{\alpha}=A d p
$$


Integrating both sides of Eq. 3, gives $A=\left(\sum_{\alpha=1}^{N_{c}} f^{\alpha}\right) / p$, considering that all the contact forces vanish at $p=0$. By substituting the value of $A$ back into Eq. 2 , we obtain the following rather intuitive form of preferential growth of forces with respect to changes in stress, i.e.

$$
\frac{d f^{c}}{f^{c}}=\frac{d p}{p}
$$

The crucial point to keep in mind here is that Eq. 4 is postulated for every single contact ( $N_{c}$ number of equations), and from this point of view, the relation in Eq. 4 is different from, but still consistent with, the Love-Weber stress averaging formula [16].

The two sides of Eq. 4 can now be integrated to find the evolution of contact forces with hydrostatic stress:

$$
f^{c}(p)=\frac{f_{0}^{c}}{p_{0}} p
$$

with $f_{0}^{c}$ being the reference value of the contact $c$ at the reference pressure $p_{0}$. The relation in Eq. 5 predicts a linear variation of contact forces with pressure in a hydrostatic loading path essentially because the coefficient of proportionality in Eq. 4 is equal to 1. Otherwise, the integration of Eq. 4 will lead to a power function for force in terms of pressure.

\subsection{DEM Simulation: Hydrostatic Stress}

The relations expressed in Eqs. 4-5 are derived based on the preferential growth hypothesis (Eq. 1) whose validity is next checked through 2D DEM simulations performed using YADE software [17]. The simulation is carried out on a square assembly of 5,000 circular particles with radii uniformly distributed (by mass percentage) between 0.75 and $1.2 \mathrm{~mm}$. A stiffness parameter, $E=10^{6} \mathrm{kN} / \mathrm{m}^{2}$ is assigned to the particles, based on which the linear normal stiffness at each contact point is calculated as $k_{n}=E \frac{2 r_{1} r_{2}}{r_{1}+r_{2}}$ where $r_{1}$ and $r_{2}$ are the radii of the particles in contact. The tangential and normal stiffnesses at contact points are assumed to be the same, $k_{t}=k_{n}$. A Coulomb friction limit of $\mu=0.5$ is used for all the contacts. To avoid overcrowding the figures and for a better readability, the results in this paper are presented in their dimensional forms with SI units.

Using periodic boundary conditions, a relatively loose assembly of particles has been brought to static equilibrium under hydrostatic pressure of $p=$ $0.01 \mathrm{kN} / \mathrm{m}$ with a $2 \mathrm{D}$ porosity of 0.167 . The hydrostatic pressure is then gradually increased to $p=1 \mathrm{kN} / \mathrm{m}$, while the evolution of contact forces was tracked.

Figure 2 shows the incremental change in the magnitude of all contact forces within the assembly due to an incremental change of $d p=0.01 \mathrm{kN} / \mathrm{m}$ applied at a current stress level of $p=0.2 \mathrm{kN} / \mathrm{m}$. The results in Fig. 2 indeed verify the accuracy of the prediction by Eq. 4 which is represented by the 1-to-1 line. The red square symbols with the error bars reflect the average variation of the 


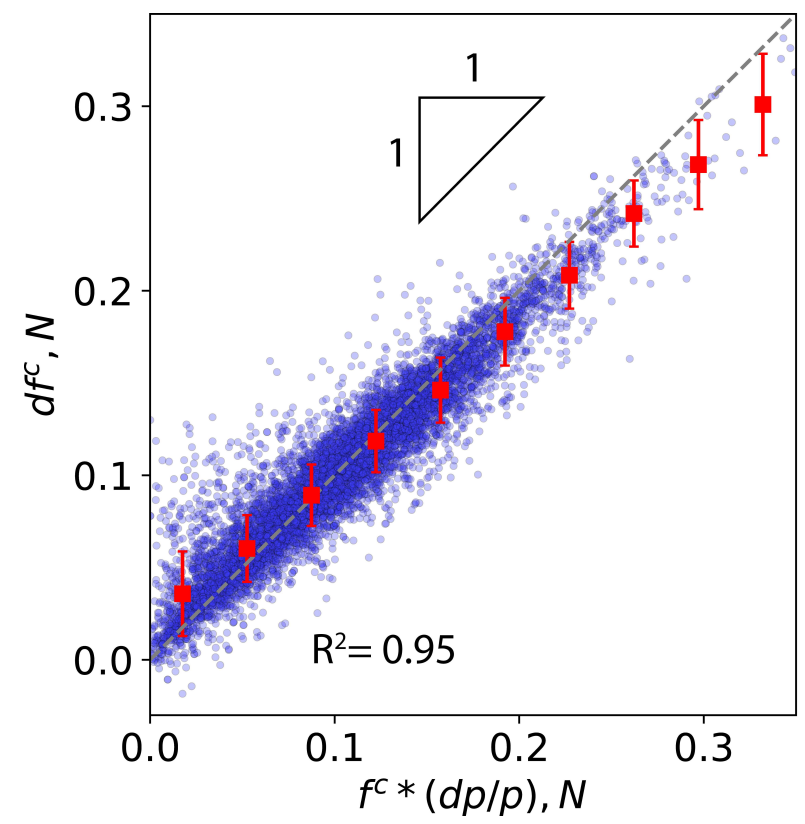

Figure 2: Incremental change in the magnitude of all the contact forces, $d f^{c}$, versus the magnitude of the contact force, $f^{c}$, scaled with a factor of $d p / p$. All the values are measured at $p=0.2 \mathrm{kN} / \mathrm{m}$ for an increment in hydrostatic stress of $d p=0.01 \mathrm{kN} / \mathrm{m}$. The 1 -to- 1 line represents the prediction by Eq. 4 . The red square symbols represent the average variation of scattered results.

results, whose trend, while still linear, exhibits a slight deviation from 1-to-1 line. The consequences of this deviation will be discussed later on.

It is worth mentioning that, given the prefatory nature of the current study, herein and thereafter, the data points are collected from a single DEM simulation and no ensemble averaging has been performed. Indeed, future studies including such ensemble averaging will better determine the confidence in the outcomes. Moreover, while not presented here, we confirm that the accuracy of the relation remains the same as the hydrostatic pressure increases and the dispersion of the points only changes with the stress increment size, $d p$.

Furthermore, Eq. 5 predicts a linear increase in the magnitude of the contact forces as the hydrostatic pressure increases, which is demonstrated by the results in Fig. 3. The inset graph shows the linear evolution predicted by Eq. 5 for five selected contacts with the match being more accurate for stronger contact forces. The slight deviation from the linear trend for weaker contact forces is most probably due to the fact that weak forces (associated with newly formed contacts) are more prone to local adjustments to satisfy equilibrium requirements.

Equation 5 also predicts that, when scaled properly, the evolution of all 
contact forces collapses into a single linear 1-to-1 trend, which is convincingly verified by the results displayed in Fig. 3. In this figure, the cloud of points represents the evolution of $f^{c}$ for all persisting contact forces throughout the hydrostatic loading path, with $f^{c}$ being the magnitude of a given contact force at a given hydrostatic pressure, $p$, while $f_{0}^{c}$ is the value of the same contact at a reference pressure $p_{0}$. The slight deviation from linearity at larger pressures observed in average values (red square symbols) is reflective of the deviation of the results in Fig. 2 from a 1-to-1 line. As explained earlier in the derivation of Eq. 5, any proportionality other than 1 in Eq. 4 will result in a non-linear variation of force with pressure.

It is also worth mentioning that the accuracy of the match between Eq. 5 and the simulation results is sensitive to the choice of reference pressure, $p_{0}$, since, at small pressures, the scaling factor $f_{0}^{c} / p_{0}$ in Eq. 5 can attain arbitrary values as the fluctuations in force values become more significant. In Fig. 3, the assembly at $p_{0}=0.2 \mathrm{kN} / \mathrm{m}$ is taken as the reference state. Also, Fig. 3 is restricted to persisting contacts simply because only the contacts that already exist at the reference pressure $p_{0}$ can be considered for evaluation of Eq. 5. For the range of pressure considered in Fig. 3, persisting contacts comprise $\sim 92 \%$ of the all the contacts.

At first glance, the existence of preferential growth for the granular assembly under hydrostatic stresses may appear to be rather trivial considering that, from a purely static point of view, for any contact force configuration at equilibrium with external stresses, the equilibrium is maintained if stresses and forces are all scaled with the same factor. However, strictly speaking, such a proportional growth in contact forces is not a-priori guaranteed in a DEM simulation which is dynamic in nature and involves a large number of internal kinematic degrees of freedom. Indeed, the scatter of the DEM data in Fig. 2 reflects the deviation from such a trivial proportional growth of the contact network. Therefore, while intuitive, the results of hydrostatic compression provide a baseline for the accuracy of preferential growth hypothesis and the degree of scatter in the results.

\section{Extension to Deviatoric Stress}

Appropriate though the hydrostatic loading case can be in demonstrating the main ideas of preferential growth and establishing a statistical baseline, it is nevertheless known among DEM practitioners that the method is often incapable of satisfactorily reproducing the intricacies of hydrostatic compaction loading as observed in experiments.

In this section, therefore, we postulate an extended version of the preferential growth that can be applied to deviatoric loading paths by considering separately the evolution of contact force components along each principal stress direction. In this case, the preferential growth in Eq. 4 is generalized to the following form:

$$
\frac{d f_{i}^{c}}{f_{i}^{c}}=\frac{d \sigma_{i}}{\sigma_{i}}
$$




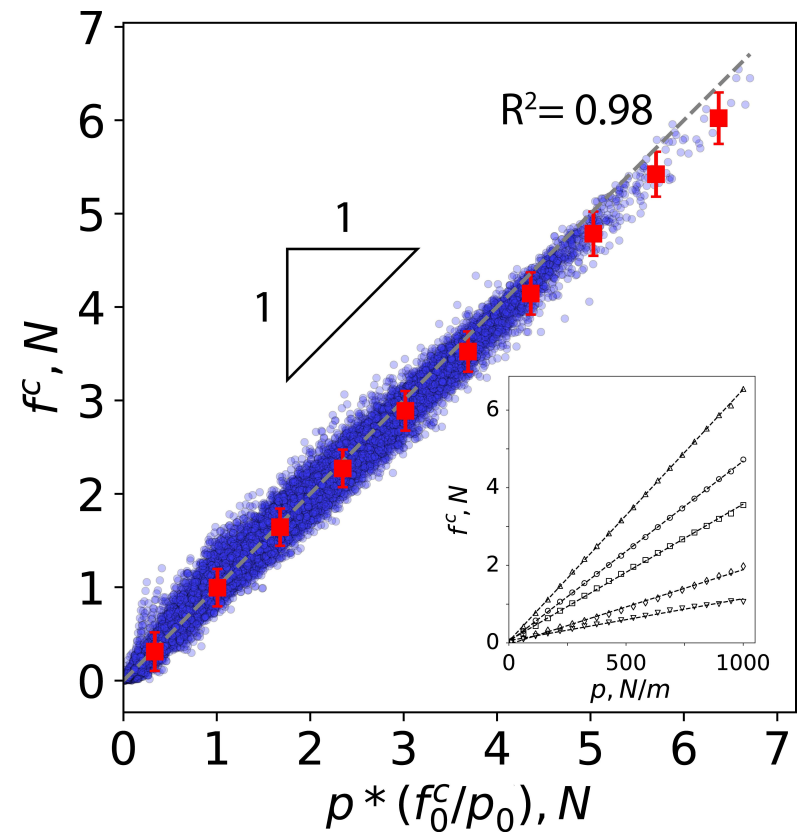

Figure 3: Accuracy of Eq. 5 (1-to-1 line) verified for all the contacts as the hydrostatic pressure $p$ is increased from $p_{0}=0.01$ to a final value of $p_{0}=1 \mathrm{kN} / \mathrm{m}$. The inset graph shows the linear increase in magnitude of five selected contacts, as the hydrostatic pressure increases. The red square symbols represent the average variation of scattered results. 
where $f_{i}^{c}$ is the component of the contact force in a frame of reference that coincides with the principal directions of the stress tensor, and $\sigma_{i}$ is the principal value of stress along the $i^{t h}$ direction. In our 2D cases with no shear stress applied at the sample level, the index $i$ refers to $x$ and $y$ directions. Equation 6 now expresses two preferential growth processes acting separately along the two principal directions, and as such, the relation can be applied to deviatoric loading paths with the two principal values of stress varying independently.

The validity of the postulate in Eq. 6 is checked through DEM simulation of an isobaric test where, starting from a hydrostatic confinement, the vertical stress, $\sigma_{y}$, is increased while the horizontal stress, $\sigma_{x}$ is decreased with the same rate such that the hydrostatic component of stress, $p=\left(\sigma_{x}+\sigma_{y}\right) / 2$, is maintained constant. In the absence of external shear stresses, the principal direction of stress are maintained along horizontal and vertical directions. The sample has been prepared in a relatively dense state with an initial porosity of 0.151 under an initial confining pressure of $1 \mathrm{kN} / \mathrm{m}$. Other simulation parameters are kept the same as the hydrostatic test described in the previous section. A denser initial state is adopted here to avoid local instabilities upon deviatoric loading. Following an isobaric (a.k.a $p$-constant) stress path, the deviatoric stress is increased until a deviatoric stress level of $q / p=\left(\sigma_{y}-\sigma_{x}\right) /\left(\sigma_{y}+\sigma_{x}\right)=0.1$ is reached. This deviatoric stress level is considerably below the peak value which occurs around $q / p=0.45$.

After reaching the desired deviatoric stress level, a small increment of stress $d \sigma_{y}=-d \sigma_{x}=0.01 \mathrm{kN} / \mathrm{m}$ is applied and the change in all the contact forces is tracked, which is compiled in Fig. 4. The horizontal axes of Fig. 4 have been scaled with the absolute value of stress changes so that the increase in $y$ direction and decrease in $x$ direction for the components of the contact forces are better distinguished. Considering the prevalence of contact gain and loss mechanisms at this stage of loading [18], only the evolution of persisting contacts is included.

Although the component-wise evolution of the contact forces in Fig. 4 is rather scattered compared to the hydrostatic case (cf. Fig. 2), nevertheless, the overall trends indeed follow the postulate in Eq. 6 for both $x$ and $y$ directions, regardless of the direction of loading. The higher dispersion of data in Fig. 4 can be explained due to the fact that force chains transmitting the vertical/horizontal stresses are not themselves strictly vertical/horizontal and as such, some degree of coupling can be expected between the vertical and horizontal components of the force and how they evolve.

Considering that the increments of stress in horizontal and vertical directions are of opposite signs, the general form of the relation in Eq. 6 can be said to be valid under deviatoric loading paths. Despite the notable scatter of data, the existence of a statistically significant 1-to-1 correlation in Fig. 4 is confirmed by narrow $95 \%$ confidence intervals for the slope of the linear fit which is calculated to be $1.0 \pm 0.066$ for Fig. 4-(top), and $1.0 \pm 0.028$ for Fig. 4-(bottom). It should be noticed that, unlike the hydrostatic loading case, the existence of a preferential growth under deviatoric loading (i.e. Eq. 6) is not obvious beforehand because, when the incremental loading deviates from the its preceding loading path, the force network evolution is not bound by mechanical considerations to follow the 

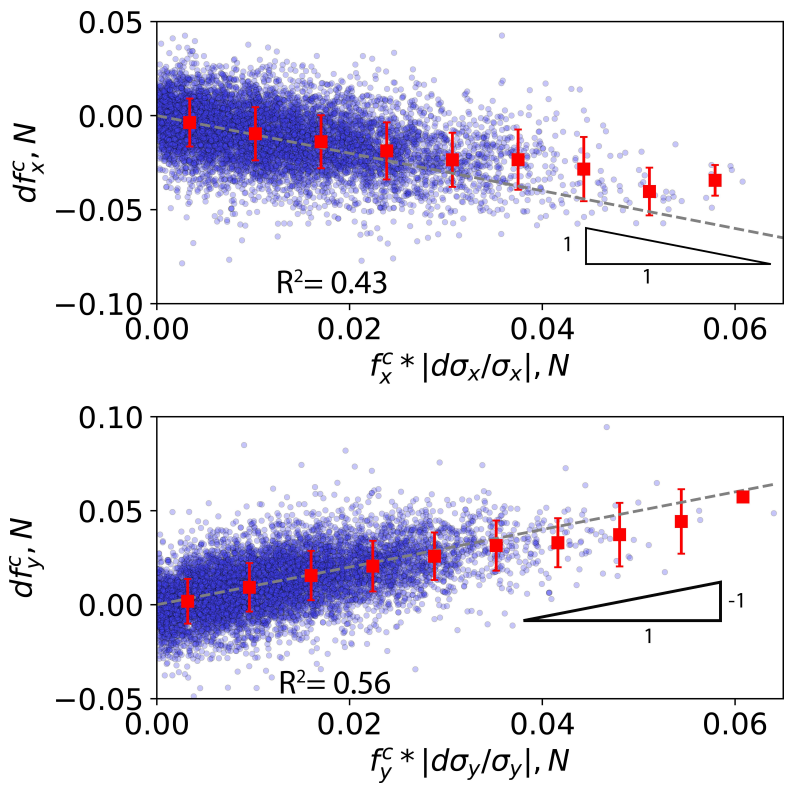

Figure 4: Incremental change in the $x$ component (top) and y component(bottom) of all the contact forces, versus their current value, $f_{x}^{c}$ and $f_{y}^{c}$, scaled with a associated stress changes. The 1-to-1 line represents the prediction by Eq. 6 . The red square symbols represent the average variation of scattered results. 
existing contact force values.

Generally speaking, it should also be kept in mind that at the limit of sliding, the evolution of contact forces can be conditioned by the frictional criterion. This issue is however avoided in the case studied herein, since the current stress state is chosen to be considerably below the peak value where the number of sliding contacts is known to be insignificant [6].

\section{Discussion and Conclusions}

The basic concept of preferential growth in contact forces explains how the heterogeneity in force distribution is accentuated as the external loading intensifies. By circumventing the specific mechanical considerations, the presence of such a preferential growth mechanism delineates one of the most prominent commonalities between granular media and other self-organized complex systems where strikingly similar internal structures are observed. Simple though it may seem in the face of it, the positive feedback mechanism associated with the preferential growth potentially entails fundamental consequences in understanding the mechanics of granular media, as briefly noted below.

- The positive feedback in preferentially growing processes is often associated with power-law distributions that are widely observed in a variety of dynamic systems [15]. This is in contrast with the probability distribution of contact forces which is known to exhibit an exponential decay, particularly for strong forces $[19,20]$. In this case, the probability distribution of contact forces in granular media can be seen to be the result of preferential growth, subjected to the requirements of static equilibrium. Qualitatively speaking, the static equilibrium condition prevents the possibility of developing extremely large forces at contacts since such forces should be in eventual static balance with other contact forces acting on the same particle. Such a requirement precludes the occurrence of a powerlaw distribution which predicts a gentler decay whereby the existence of extremely large forces is not impossible. The conclusion herein is in accordance with the results from simple lattice models where a power-law distribution is only observed for the unrealistic cases [21]. Nonetheless, the scaling of the force distribution with the average force can indeed be explained through the preferential growth process which preserves the exponential functional form as the ratio between different contact forces is conserved.

- Inspired by multiphase mechanics, the homogenization methods for granular material is often based upon the so-called Hill's microhomogeneity condition, whereby the average of local products of stresses and strains is considered to be equal to the product of average stresses and strains [22]. Therefore, from a statistical point of view, Hill's lemma requires the local values of stresses and strains to be statistically independent. By contrast, the preferential growth inherently expresses a clear statistical dependency 
between the current value of forces and their incremental change, with the former being related to current stress and the latter to the deformation at contacts through a stiffness parameter, and hence to the strain. Consequently, a dependency between the current forces and their increment can potentially lead, contrary to Hill's lemma requirement, to a dependency between local stress and strain increments. While the argument is far from being conclusive, the existence of preferential growth process can nevertheless shed new light on the ongoing debate about the interpretation of Hill's lemma for granular media [23, 24].

- The results of this study demonstrate preferential growth to be among the key processes governing the evolution of a granular force network. Turning to the spatial distribution of forces and stress, it turns out that in granular media the positive feedback associated with preferential growth acts the opposite way to diffusion mechanisms. As such, while other processes naturally exist in conjunction with preferential growth, one cannot expect the spatial distribution of stress in confined granular materials to be explicable in terms of merely diffusive processes and their associated parabolic spatial distributions. This is in agreement with the results in [25] where the parabolic forms of stress distributions, as predicted by diffusion-like processes, are ruled out in favour of hyperbolic or elliptic forms.

The points raised above touch upon ramifications of the preferential growth concept that enhance the interpretation and understanding of distinct characteristics in granular mechanics. This brief communication, nevertheless, does not claim to have yet provided definite answers to these fundamental questions. Indeed, deeper interpretations of the above conjectures require rigorous analyses and simulations that revisit such mechanical concepts in the light of the dependency between the current state and the distribution of the applied increments, as expressed by the preferential growth postulate. In particular, the extended form of Eq. 6 should be checked for general loading directions in order to better distinguish between the evolution in closed stress loading cycles. As

it stands, the preferential growth is not necessarily expected to be validated if the direction of incremental loading deviate from its preceding loading path.

Of interest, moreover, can be the application of the preferential growth framework to the evolution of forces in later loading regimes where local instabilities in the form of microavalanches prevail.

\section{Acknowledgements}

This work is a modest tribute to the legacy Bob Behringer has left during his quest to understand the origin of force distribution in granular materials over the intervening years. It is also the fruit of a long-standing collaboration between the Calgary/Grenoble/IRSTEA research groups. Research support from the Natural Science and Engineering Research Council of Canada (Grant \# STPGP 462988) is gratefully acknowledged. 


\section{Compliance with Ethical Standards}

Conflict of interest: The authors declare that they have no conflict of interest.

\section{References}

[1] P. Dantu, Proc. 4th ICSMFE, London, 1957 (1957)

[2] A. Drescher, G.D.J. De Jong, Journal of the Mechanics and Physics of Solids 20(5), 337 (1972)

[3] P.A. Cundall, O.D. Strack, Geotechnique 29(1), 47 (1979)

[4] T.S. Majmudar, R.P. Behringer, Nature 435(7045), 1079 (2005)

[5] D. Howell, R. Behringer, C. Veje, Physical Review Letters 82(26), 5241 (1999)

[6] M. Pouragha, R. Wan, Granular Matter 18(3), 1 (2016)

[7] D. Helbing, H.J. Herrmann, M. Schreckenberg, D.E. Wolf, Traffic and granular flow'99: social, traffic, and granular dynamics (Springer Science \& Business Media, 2012)

[8] A.L. Barabási, R. Albert, Science 286(5439), 509 (1999)

[9] F. Eggenberger, G. Pólya, ZAMM-Journal of Applied Mathematics and Mechanics/Zeitschrift für Angewandte Mathematik und Mechanik 3(4), $279(1923)$

[10] G. Udny Yule, Philosophical Transactions of the Royal Society of London Series B 213, 21 (1925)

[11] R.K. Merton, Science 159(3810), 56 (1968)

[12] A.H. Clark, A.J. Petersen, L. Kondic, R.P. Behringer, Physical review letters 114(14), $144502(2015)$

[13] M. Pouragha, Continuum representation of the micromechanics of granular materials via homogenization and statistical approaches (chapter 7). Ph.D. thesis, University of Calgary (2015)

[14] M. Pouragha, R. Wan, Mechanics of Materials 126, 57 (2018)

[15] R. Albert, A.L. Barabási, Reviews of modern physics 74(1), 47 (2002)

[16] J. Weber, Bulletin de Liaison des Ponts et Chaussées 20, 3 (1966)

[17] V. Šmilauer, E. Catalano, B. Chareyre, S. Dorofeenko, J. Duriez, A. Gladky, J. Kozicki, C. Modenese, L. Scholtès, L. Sibille, et al., Yade Documentation 474, 1 (2010) 
[18] M. Pouragha, R. Wan, International Journal of Solids and Structures 110, 94 (2017)

[19] H.M. Jaeger, S.R. Nagel, R.P. Behringer, Reviews of modern physics 68(4), 1259 (1996)

[20] D. Mueth, H. Jaeger, S. Nagel, Physical Review E 57(3), 3164 (1998). DOI 10.1103/PhysRevE.57.3164

[21] S. Coppersmith, C.h. Liu, S. Majumdar, O. Narayan, T. Witten, Physical Review E 53(5), 4673 (1996)

[22] R. Hill, Proc. R. Soc. Lond. A 326(1565), 131 (1972)

[23] F. Calvetti, G. Combe, J. Lanier, Mechanics of Cohesive-frictional Materials: An International Journal on Experiments, Modelling and Computation of Materials and Structures 2(2), 121 (1997)

[24] D. Caillerie, in International Workshop on Bifurcation and Degradation in Geomaterials (Springer, 2017), pp. 467-473

[25] R. Blumenfeld, Physical review letters 93(10), 108301 (2004) 